\title{
Aplicación móvil para la enseñanza de lesiones elementales en cavidad bucal
}

\author{
A mobile application for teaching elementary lesions in the oral cavity \\ Eilien Tovio-Martínez ${ }^{*}$ orcid.org/0000-0003-3702-2791 \\ Martha Carmona-Lordouy ${ }^{1}$ orcid.org/0000-0003-3066-2219 \\ Jonathan Harris ${ }^{1}$ orcid.org/0000-0002-4662-0729 \\ Eliana Guzmán1 orcid.org/0000-0002-8475-6301
}

1 Facultad de Odontología, Universidad de Cartagena, Cartagena, Colombia

\section{Resumen}

Introducción: El desarrollo de las Tecnologías de la Información y la Comunicación (TIC) y su fácil acceso, hacen de ésta una alternativa educativa que contribuye a incrementar la calidad de la educación, facilitando el proceso de enseñanzaaprendizaje con medios virtuales. Las lesiones elementales son alteraciones del tejido bucal y su conocimiento es importante para realizar un adecuado diagnóstico y manejo terapéutico. Objetivo: Diseñar, implementar y evaluar una aplicación móvil para la enseñanza de lesiones elementales en cavidad bucal. Materiales y métodos: Estudio de intervención, experimental, que evaluó una herramienta educativa sobre lesiones elementales bucales, utilizando una preprueba, una intervención y una prueba posterior, para el grupo control se evaluó el aprendizaje a través de la lectura de un artículo. Se compararon los resultados obtenidos mediante prueba de $\mathrm{Ch}^{2}$ con un $p \leq 0,05$. Resultados: Ambos grupos lograron un incremento en el nivel de conocimiento sobre lesiones elementales; sin embargo, quien utilizó la aplicación móvil obtuvo resultados significativamente mejores de aprendizaje $\left(C h i^{2} p=0,014\right)$. Conclusiones: Las TIC constituyen una estrategia útil para afianzar nuevos conocimientos, se comprobó que los estudiantes que emplearon la herramienta educativa incrementaron significativamente el aprendizaje en lesiones elementales de una forma agradable y amena.

Palabras clave: Boca; educación en salud; tecnología de la información; patología bucal; aprendizaje; efectividad. (Fuente: DeCS, Bireme).

\begin{abstract}
Introduction: The development and widespread access to Information and Communication Technologies (ICTs) have reinforced the view of them as didactic alternatives to improve the quality of education. These technological advances have facilitated the teaching-learning process through the use of virtual media. Knowing elementary lesions in the oral cavity tissue is important for their appropriate diagnosis and therapeutic management. Objective: To design, implement and assess the use of a mobile application in the teaching of elementary lesions of the oral cavity. Materials and methods: An intervention and experimental study that evaluated a didactic tool about oral elementary lesions, applying intervention, pre and post testing. The control group learning skills were assessed through the reading of an article. We finally compared the results via $\mathrm{Chi}^{2}$ test, with $p \leq 0.05$. Results: Although both groups showed an improvement in learning levels, the group that used the mobile application achieved significantly better results in reference to learning about elementary lesions (Chi ${ }^{2}$ $p=0.014$ ). Conclusions: ICTs have become useful strategies to strengthen new knowledge. In this study, we show that students who used the educational tool improved the learning of elementary lesions, in a significant and enjoyable way.
\end{abstract}

Key words: Mouth; health education; information technology; oral pathology; learning; effectiveness. (Source: DeCS, Bireme).

\footnotetext{
*Autor de correspondencia

Eilien G. Tovío Martínez

e-mail: etoviom@unicartagena.edu.co
} 


\section{Introducción}

Las tecnologías de la información y la comunicación (TIC) son todos los medios desarrollados a partir de la informática, que permiten la comunicación e interacción con fines educativos; de manera sincrónica o asincrónica, o colectiva, desde lugares geográficamente distantes(1). Existe un vínculo entre la educación y las TIC que toma fuerza con el pasar del tiempo. Actualmente, la formación básica, media y superior buscan la manera de utilizar esta unión para mejorar la cobertura y facilitar los procesos pedagógicos ${ }^{(2)}$. A pesar de los beneficios que brindan las TIC, estas pueden ser usadas de forma inapropiada para difundir información equívoca que puede generar inconvenientes en el aprendizaje y en el ejercicio de la profesión; por esta razón, es importante formar a las personas en el uso de las nuevas tecnologías para que estas herramientas no se conviertan en un recurso subexplotado(3).

En la actualidad se evidencia la utilidad y la interacción que existe entre los avances tecnológicos y la educación, permitiendo a los estudiantes y docentes definir nuevos roles y utilizar estos medios como estrategias didácticas que contribuyan a un mejor desempeño y rendimiento académico. Los dispositivos electrónicos como tablets, móviles, iPad, se convierten en una herramienta de trabajo que puede ser utilizada para transformar los conocimientos ya establecidos y darles otros usos, permitiendo que el estudiante adquiera mayor autonomía y responsabilidad en el proceso de aprendizaje y obligando al docente a salir de su rol clásico como única fuente de conocimiento; esto lleva a una reestructuración creativa de la institución escolar $(4,5)$.

El incremento en la implementación de las TIC en el aula de clase ayuda a los estudiantes en el aprendizaje, cumpliendo un rol de transmisión de saberes, desarrollo de habilidades y destrezas en las tecnologías ya creadas; por ello, las instituciones educativas enfrentan un gran reto que demanda a los docentes establecer estrategias basadas en la apropiación de los procesos tecnológicos, aplicándolas en cada ámbito profesional o técnico; apoyándose en diferentes elementos para la investigación y la innovación de los procesos, productos y servicios provenientes de las áreas tecnológicas(6).Las lesiones elementales son indicadores o signos clínicos, que se manifiestan en la mucosa bucal y se caracterizan por presentar cambios en la forma, tamaño, textura, consistencia, color y relieve. Estas alteraciones pueden aparecer sobre una mucosa sana, llamándose lesiones elementales primitivas o primarias, por el contrario, si estas lesiones derivan de las primarias, o son consecuencia de la evolución de una lesión primaria, se denominan lesiones elementales secundarias. Es necesario su adecuado reconocimiento para brindar un diagnóstico y tratamiento oportuno a cada paciente(7).

La mucosa bucal tiene un número limitado de respuestas patológicas, éstas se conocen como lesiones elementales y son capaces de caracterizar a las enfermedades mucocutáneas, produciéndose como consecuencia de agresiones por agentes externos, o bien, son la manifestación de una enfermedad sistémica, las causas son el trauma ocasionado por prótesis, su higiene, la reacción irritante y alergia al material en la elaboración de la misma, además de factores dietéticos, infecciones por microorganismos y factores sistémicos(8). Estos signos clínicos constituyen los principios en los cuales se sustenta el lenguaje y el pensamiento dermatológico y estomatológico, siendo semejante al alfabeto sobre el que se fundamenta el sistema de comunicación. En la práctica estomatológica, es de vital importancia el conocimiento y la interpretación del significado de cada una de las lesiones elementales, permitiendo una correcta asociación entre lesiones y su imagen respectiva. Al identificar las imágenes de la enfermedad y los datos recolectados en la anamnesis se puede obtener diagnósticos oportunos, tratamientos efectivos y eficaces en cada patología oral(9).

Frente a la cantidad de alteraciones que se pueden encontrar en la mucosa bucal, los odontólogos deben ser capaces de detectarlas para llevar a cabo un correcto diagnóstico y apropiado tratamiento. Una conducta inadecuada al realizar la exploración convencional de la mucosa oral y al establecer el diagnóstico diferencial de las lesiones puede suponer el pasar por alto lesiones importantes o el indicar un tratamiento incorrecto(10). El propósito de este artículo es diseñar, implementar y evaluar una aplicación móvil para identificación y enseñanza de lesiones elementales en cavidad bucal, en estudiantes del Programa de Odontología de la Universidad de Cartagena, permitiendo el manejo de las TIC como estrategia de aprendizaje. 


\section{Materiales y métodos}

Se realizó un estudio de intervención, de naturaleza experimental, de tipo intervención educativa, utilizando una metodología que incluyó una preprueba, una intervención y una prueba posterior, así como un grupo de control. La muestra estuvo constituida por 74 estudiantes (37 para el grupo intervención y 37 para el grupo de control) pertenecientes al Programa de Odontología de la Universidad de Cartagena, que cursaron el primer periodo del 2017 en los ciclos académicos de VIII, IX y X semestre, quienes se encontraban nivelados con sus materias, participaron voluntariamente en el estudio y que tenían equipos móviles con Sistema Android.

\section{Fase 1: Diseño de la aplicación móvil}

Se diseñó y creó una aplicación móvil como herramienta educativa, la cual se instauró como guía diagnóstica de patologías orales a partir de lesiones elementales, fundamental para la formación integral de estudiantes en proceso de formación odontológica. Esta aplicación se realizó en compañía de un ingeniero de sistemas capacitado durante un tiempo aproximado a 4 meses, en donde se hizo una exhaustiva revisión bibliográfica, la selección fotográfica de cada lesión elemental en conjunto con su patología y la realización de videos para la explicación de las temáticas relacionadas con el diagnóstico de patologías bucales.

\section{Fase 2: Asignación aleatoria}

Antes de dar a conocer la aplicación a los estudiantes se realizó la asignación aleatoria a los dos grupos, G1: grupo intervención (recibieron información de la aplicación móvil). G2: grupo control (se entregó información impresa de la temática). Se realizó la asignación aleatoria a uno de los dos grupos intervención y control, utilizando el aplicativo para tal fin del Software Epidat versión 4.1.

\section{Fase 3 y 4: Implementación y evaluación de la aplicación (técnicas e instrumentos para recolección de información)}

La implementación consistió en la instalación de la aplicación en los dispositivos con una demostración sólo al grupo de intervención. Se hizo una evaluación inicial de 1 hora (pre-test), con un cuestionario de 10 preguntas de selección múltiple, que permitía identificar cada lesión, y establecer las diferencias entre lesiones y la asociación de cada lesión con la patología respectiva. La calificación fue cuantitativa en una escala de 1 a 5 ; después se realizó la instalación de la aplicación en los diferentes dispositivos con sistema operativo Android de los estudiantes del subgrupo 1 , mientras que a los estudiantes del subgrupo 2 se les entregó copias de un documento relacionado con el tema. Una vez implementadas las diferentes estrategias educativas (aplicación móvil Vs método tradicional), se aplicó el segundo instrumento de evaluación 8 días después de la primera intervención (pos-test), para comparar la eficiencia de ambas estrategias y valorar el nivel de percepción de la aplicación móvil en los usuarios. Además se aplicó una encuesta al subgrupo 1, que evaluó la efectividad de la aplicación móvil y el nivel de satisfacción con la herramienta educativa. Finalmente, se procedió a calificar los test y digitar los datos obtenidos en una base de Excel; a cada participante se le asignó un código.

\section{Fase 5: Comparación de los resultados de la aplicación (interpretación de datos)}

Para el análisis de variables cualitativas se calculó medidas de frecuencia y proporciones e intervalo de confianza del 95\%. Si los datos seguían una distribución normal, se reportaba la media y desviación estándar, en caso contrario mediana y rango intercuartílico.

Para la comparación de los niveles de conocimiento de acuerdo a grupos de estudio, si los datos presentaban una distribución normal, se empleaba la prueba de $t$ de Student no pareada; si no, pruebas estadísticas no paramétricas, como $\mathrm{Chi}^{2}$ considerando el nivel de significancia estadística $p<0,05$. Para la construcción de la base de datos se trabajó con el software estadístico Stata 11.0.

\section{Consideraciones éticas}

Todo el proceso realizado en este estudio se rigió por la Resolución 8430 de 1993 del Ministerio de Salud y Protección Social de Colombia y la Declaración de Helsinki, donde se catalogó como un estudio sin riesgo, contando con el aval por parte del Comité de Ética en investigaciones de la Facultad de Odontología, de la Universidad de Cartagena. Asimismo, se tuvo en cuenta la firma del consentimiento informado por parte de cada uno de los estudiantes. 


\section{Resultados}

De un total de 74 estudiantes, el 59,46\% (44) correspondían al género femenino, y el 40,54\% (30) al género masculino, el 37,84\% (28) pertenecían a noveno semestre, $33,78 \%$ (25) a décimo y $28,38 \%$ (21) a octavo semestre, el promedio de edad de los estudiantes fue de 23,72 $\pm 3,49$ años (Tabla 1).

A nivel general, al realizar la evaluación inicial o pretest, se obtuvo un nivel de conocimiento alto en el $33,8 \%$ (25) de los estudiantes, el 63,5\% (47) nivel medio y el 2,7\% (2) bajo. Luego de las intervenciones se realizó una segunda evaluación donde se encontró un nivel de conocimiento alto con un $64,9 \%$ (48), seguido de nivel medio $32,4 \%$ (24) y bajo en un 2,7\% (2). Los datos obtenidos fueron procesados y tabulados, se realizó el análisis, en donde el valor estadístico fue determinado por medio del $C h i^{2}$, con un nivel de significación $p<0,05$, el cual permitió encontrar diferencias estadísticamente significativas $(p=0,016)$.

Al comparar los dos tipos de intervenciones educativas, método tradicional (lectura) vs. aplicación móvil, los estudiantes que fueron asignados a la aplicación (37) en la evaluación inicial o pre-test, tenían un nivel de conocimiento alto representando el 42,1\% (16), el 52,6\% (19) tuvo nivel regular y el 5,3\% (2) bajo. Luego de las intervenciones se destaca un nivel de conocimiento alto con el 71,1\% (26); el 26,3\% (10) medio y el 2,6\% (1) bajo. El análisis se realizó usando $\mathrm{Chi}^{2}$ sin encontrar diferencias estadísticamente significativas $(p>0,05)$.

Con respecto a los estudiantes que estuvieron asignados al grupo de estudio con el método tradicional (37), en el pre-test, en el nivel de conocimiento sobre lesiones elementales, el 25\% (9) obtuvo un nivel alto y el 75\% (28) un nivel regular, ninguno de los participantes obtuvo nivel de conocimiento bajo. Luego de la intervención con el método tradicional el 58,3\% (22) alcanzó un nivel alto; el 38,9\% (14) fue medio y el 2,8\% (1) bajo. El análisis se efectuó a través de la prueba estadística $\mathrm{Chi}^{2}$, encontrando diferencias estadísticamente significativas $(p=0,014)$ (Tabla 2). De los 74 participantes, sólo aquellos que estudiaron utilizando la aplicación (50\%), fueron quienes evaluaron los aspectos relacionados con la encuesta de satisfacción, sin embargo un estudiante no respondió dicha encuesta quedando 36 estudiantes. En la encuesta de satisfacción, para el ítem de efectividad, el $4,1 \%$ (3) estuvo totalmente desacuerdo, el 2,7\%(2) desacuerdo, el 1,4\% (1) estuvo indeciso, el 27\% (20) estuvo de acuerdo que la aplicación fue efectiva, seguido de 14,8\% (10) totalmente de acuerdo (Tabla 3).

Tabla 1. Características sociodemográficas población de estudio

\begin{tabular}{|c|c|c|c|c|c|c|c|c|c|}
\hline & \multicolumn{3}{|c|}{ Globales } & \multicolumn{3}{|c|}{ Aplicación } & \multicolumn{2}{|c|}{ Copias } & \multirow{2}{*}{ Valor de $p$} \\
\hline & $n=74$ & $\%$ & & n(37) & $\%$ & & n (37) & $\%$ & \\
\hline Género & & & & & & & & & \multirow{3}{*}{$0,506 ¥$} \\
\hline Masculino & 30 & & 40,5 & 13 & & 36,8 & 17 & 44,4 & \\
\hline Femenino & 44 & & 59,5 & 24 & & 63,2 & 20 & 55,6 & \\
\hline \multicolumn{10}{|l|}{ Semestre } \\
\hline VIII & 21 & & 28,4 & 12 & & 31,6 & 10 & 25,0 & \multirow{3}{*}{$0,757 ¥$} \\
\hline IX & 28 & & 37,8 & 12 & & 31,6 & 15 & 41,7 & \\
\hline $\mathrm{X}$ & 25 & \multirow{3}{*}{ DE } & 33,8 & 13 & & 36,8 & 12 & 33,3 & \\
\hline \multirow[t]{2}{*}{ Edad } & Media & & & Media & DE & & Media & DE & Valor de $p$ \\
\hline & 23,7 & & 3,5 & 23,50 & & 2,43 & 23,94 & 4,36 & $0,588 \S$ \\
\hline
\end{tabular}

$¥: C h i^{2}$. §: $t$ de Student

Tabla 2. Nivel de conocimiento sobre lesiones elementales, en la muestra estudiada antes y después de las intervenciones

\begin{tabular}{|c|c|c|c|c|c|c|c|c|c|c|c|c|c|c|c|}
\hline \multirow{3}{*}{ Nivel de conocimiento } & \multicolumn{4}{|c|}{ Global } & \multirow{3}{*}{$\begin{array}{l}\text { Valor } \\
\text { de } p\end{array}$} & \multicolumn{4}{|c|}{ Aplicación } & \multirow{3}{*}{$\begin{array}{l}\text { Valor } \\
\text { de } p\end{array}$} & \multicolumn{4}{|c|}{ Copias } & \multirow{3}{*}{$\begin{array}{c}\text { Valor } \\
\text { de } p\end{array}$} \\
\hline & \multicolumn{2}{|c|}{ Pre-test } & \multicolumn{2}{|c|}{ Post-test } & & \multicolumn{2}{|c|}{ Pre-test } & \multicolumn{2}{|c|}{ Post-test } & & \multicolumn{2}{|c|}{ Pre-test } & \multicolumn{2}{|c|}{ Post-test } & \\
\hline & n (74) & $\%$ & n (74) & $\%$ & & n(37) & $\%$ & n(37) & $\%$ & & n(37) & $\%$ & n(37) & $\%$ & \\
\hline Alto & 25 & 33,8 & 48 & 64,9 & & 16 & 42,1 & 26 & 71,1 & & 9 & 25 & 22 & 58,3 & \\
\hline Regular & 47 & 63,5 & 24 & 32,4 & $0,016 ¥$ & 19 & 52,6 & 10 & 26,3 & $0,133 ¥$ & 28 & 75 & 14 & 38,9 & $0,014 ¥$ \\
\hline Bajo & 2 & 2,7 & 2 & 2,7 & & 2 & 5,3 & 1 & 2,6 & & & & 1 & 2,8 & \\
\hline
\end{tabular}


Tabla 3. Nivel de satisfacción sobre lesiones elementales, luego de la intervención con la aplicación

\begin{tabular}{lrrrrr}
\hline & $\begin{array}{c}\text { Total desacuerdo } \\
\text { n (\%) }\end{array}$ & $\begin{array}{c}\text { Desacuerdo } \\
\text { n (\%) }\end{array}$ & $\begin{array}{c}\text { Indeciso } \\
\text { n (\%) }\end{array}$ & $\begin{array}{c}\text { De acuerdo } \\
\text { n (\%) }\end{array}$ & $\begin{array}{c}\text { Total de acuerdo } \\
\text { n (\%) }\end{array}$ \\
\hline Efectividad & $3(4,1)$ & $2(2,7)$ & $1(1,4)$ & $20(27)$ & $10(14,8)$ \\
Eficiencia & $2(2,7)$ & - & & $17(23,5)$ & $17(23,5)$ \\
Satisfacción & $5(6,8)$ & $25(35,1)$ & $6(8,1)$ & - & - \\
Facilidad de aprendizaje & $2(2,7)$ & $16(21,6)$ & $18(25,7)$ & - & - \\
Memorabilidad & $2(2,7)$ & & $1(1,4)$ & $20(27)$ & $13(18,6)$ \\
Errores & $4(5,4)$ & $20(27)$ & $6(8,1)$ & $3(4,1)$ & $3(4,1)$ \\
Contenido & $2(2,7)$ & $1(1,4)$ & $33(45,9)$ & - & - \\
Accesibilidad & $2(2,7)$ & $5(6,8)$ & $29(40,5)$ & - & - \\
Seguridad & $12(16,2)$ & $17(24,3)$ & $1(1,4)$ & $2(2,7)$ & $4(5,4)$ \\
\hline
\end{tabular}

\section{Discusión}

Existe un nexo muy importante entre la ciencia, la educación y la tecnología, que demuestra el impacto que tienen las TIC en el proceso de aprendizaje del estudiante. Tienen una incidencia a nivel de la comunidad escolar, especialmente a nivel de los docentes quienes están cada día más instruidos en las nuevas tecnologías, convirtiéndolas en un complemento de las clases presenciales que permite acceder a la información, comunicarse, debatir temas, entre otros(11).

En estudios epidemiológicos donde han sido utilizadas las TIC se ha demostrado que a través de esta herramienta se logra alcanzar el saber, ciencia y competencias de forma didáctica, convirtiéndose así en un método cautivante en la adquisición de conocimientos $^{(12)}$. Arriaga et al. en el 2018 realizaron una investigación, con una muestra de 27 estudiantes de Tecnologías de la Comunicación y Gestión de la Información (TCGI) en la Universidad Autónoma de Nayarit (UAN), al comparar los resultados obtenidos para el pre-test, se alcanzó un $62,02 \%$ de conocimiento sobre los diferentes temas que se expusieron, mientras que un $71,85 \%$ del postest, tuvo un incremento en el aprovechamiento de $9,83 \%$, evidenciando una mejora en el logro de las competencias establecidas en el uso de aplicaciones de la Web 2.0 para la evaluación del aprendizaje significativo(13), coincidiendo con el presente estudio.

En el estudio realizado por Escobar et al., en el 2015, se diseñó un objeto y un laboratorio virtual de química para la enseñanza de la ley de conservación de la masa. En este participaron 17 estudiantes del grado décimo de una Institución Educativa del municipio de Pasto. El 1,76\% de los estudiantes respondieron adecuadamente el pre-test, cifra que incrementó en el pos-test a $82,35 \%{ }^{(14)}$. Resultados similares a los encontrados en el presente estudio en cuanto al rendimiento de los estudiantes, ya que se obtuvo un nivel de aprendizaje alto luego de la intervención, evidenciando mejores notas en el posttest que en el pre-test.

Salgado et al., en 2015, comentan la aceptación del uso de las nuevas tecnologías, sin embargo se evidencia inconformidad con la plataforma y los recursos disponibles, ya que se requiere tener acceso a internet cada vez que se requiera la búsqueda de material de estudio, además existe personal se le hace difícil el manejo de las herramientas tecnológicas, lo que les lleva a pensar que la tecnología podría o no ser para los estudiantes importante, sin dejar a un lado la estrategia educativa de los libros, los cuales están siempre disponibles, a la mano del lector, son manejables y trasportables con facilidad, poseen una credibilidad alta, larga vida y cumplen un papel muy importante en el estudio de estudiantes y docentes lo cual es válido para su aprendizaje(15). Arévalo et al., en 2017, refieren que para el ámbito estudiantil, la ayuda de los libros es poco atractivo, no son interactivos y muchas veces no resuelven por completo las dudas generadas, mientras que las ayudas tecnológicas evolucionan cada vez más y están presentes en todos los ámbitos(3). Sin embargo, cabe mencionar que el grupo de estudio con el método tradicional (copias) también se presentó incremento en el aprendizaje.

Actualmente el acceso a los recursos tecnológicos permite que los estudiantes tengan un mayor grado de conectividad a internet a través de los dispositivos electrónicos sea en sus hogares, escuelas o en cualquier lugar que permita conexión a la red. Sin embargo estos recursos podrían representar un obstáculo para la integración de las TIC a la educación, ya sea por la escasez de 
ordenadores, programas educativos virtuales en las aulas de clase o conexión inestable de internet; hecho que restringe el acceso de los docentes a las tecnologías, esto conlleva de cambios de rol hacia un modelo más flexible para el sistema de enseñanzaaprendizaje. La educación evoluciona y se adapta a las exigencias de las nuevas generaciones, pasó de ser una cultura analógica del papel, lápiz, libro, pizarra, para transformarse en entornos digitales de redes, plataformas de trabajo, libros digitales y lenguajes tipo texto, numérico, icónico, visual y sonoro(16).

Díaz et al., sugieren que el aprendizaje mediante herramientas tecnológicas con el objetivo de fomentar las habilidades de estudio autónomo debe estar regulado por ciertas funciones como son, valoración de metas, monitoreo o toma de notas(10). Concordando con el actual estudio, en el que la aplicación es solo una ayuda para el conocimiento de los estudiantes, se deben mejorar las aplicaciones para que sean aprovechadas al máximo desde el punto de vista del software, pues el $8,1 \%$ de los usuarios en el presente estudio hacían uso de teléfonos móvil con sistema operativo diferente al android, lo que no permite hacer uso de la aplicación creada en específico, para que de esta manera se permita mejor adaptabilidad, rápido acceso y fácil manejo al momento de utilizarlo, independientemente del terminal del usuario.

Las instituciones académicas que forman profesionales del área de la salud son beneficiadas a través de estos estudios, ya que por medio de las ayudas didácticas se puede brindar y evaluar el conocimiento de los estudiantes y así, tomar decisiones pertinentes en cuanto a la elección de la metodología de enseñanza(17). Por medio de los resultados obtenidos se pudo responder la hipótesis que se planteó acerca de la utilidad que prestan las TIC, en este caso a través de las aplicaciones móviles, en la enseñanza por parte de los docentes, como en el aprendizaje por parte de los estudiantes, dentro y fuera del aula de clase, dando a conocer que el nivel de conocimientos sobre lesiones elementales antes de realizar la implementación educativa a los estudiantes de VIII, IX y X semestre de la Facultad de Odontología de la Universidad de Cartagena, fue medio y posterior a la implementación educativa fue alto. Córdoba et al., en 2016 comentan que al emplear las tecnologías como herramientas didácticas de apoyo, se obtiene un impacto positivo, con respecto al proceso de enseñanza y el aprendizaje(18), corroborando que las TIC permiten acercar el desarrollo de las competencias de los estudiantes a las dinámicas de hoy en día, en donde los resultados expuestos en este estudio, muestran que el nivel de aprendizaje mejoró después de la aplicación.

\section{Conclusiones}

El nivel de conocimientos sobre lesiones elementales antes de realizar la implementación educativa que predominó en los estudiantes de VIII, IX y X de odontología de la Universidad de Cartagena fue regular, mientras que posterior a ésta, el nivel predominante fue alto. Haciendo énfasis en las estrategias educativas utilizadas, se observó diferencias estadísticamente significativas; aunque en ambas permitieron adquirir un mayor nivel de conocimiento sobre lesiones elementales, se demostró que la intervención con aplicación móvil presentó mejores resultados, concluyendo que además de lograr mayores niveles de conocimiento, puede complementar un diagnóstico clínico.

El conocimiento sobre lesiones elementales es de fundamental importancia ya que gracias a ellas se puede hacer un diagnóstico precoz de patologías ubicadas en la cavidad bucal y de esta forma mejorar la calidad de vida de los usuarios.

\section{Recomendaciones}

Teniendo en cuenta que la comunidad estudiantil actualmente es cada vez más tecnológicamente alfabetizada, se hace necesario que el sistema educativo sea atractivo para mejorar la comprensión y aprendizaje en todas las áreas del conocimiento. De esta manera es imperativo y se requiere llevar a cabo el diagnóstico correcto y temprano de los diferentes tipos de lesiones que se presentan a nivel de cavidad bucal; por ello, se evidencia la importancia fundamental que existe en la vinculación de las lesiones elementales y las nuevas tecnologías, ya que gracias a ellas se puede hacer un diagnóstico precoz de patologías ubicadas en la cavidad bucal permitiendo mejorar la calidad de vida en las personas que acuden a una consulta estomatológica buscando una solución a su inconformidad.

Es pertinente seguir realizando nuevos estudios que permitan determinar el nivel de satisfacción recibido 
por parte de los estudiantes en cuanto a la implementación de las TIC en la educación.

\section{Agradecimientos}

A los docentes y estudiantes de la Facultad de Odontología de la Universidad de Cartagena que participaron e hicieron posible el desarrollo de la investigación.

Conflicto de intereses: No se declaran conflictos de intereses con relación a esta investigación.

\section{Referencias}

1. Nolasco P, Ojeda M. La evaluación de la integración de las TIC en la educación superior: fundamento para una metodología. RED-Revista de Educación a Distancia. 2016;48(9):1-24. Disponible en: https://revistas.um.es/red/article/view/253511/191301

2. Hernández-Sánchez J. Uso e integración de las TIC en dos servicios de salud de Bienestar Universitario. Revista Hacia la Promoción de la Salud. 2014;19(1):99-113. Disponible en: http://www.scielo.org.co/pdf/hpsal/v19n1/v19n1a08.pdf

3. Arévalo J, Mirón J. Aplicaciones móviles en salud: potencial, normativa de seguridad y regulación. Revista Cubana de Información en Ciencias de la Salud (ACIMED). 2017;28(3):1-13. Disponible en: http://scielo.sld.cu/scielo.php?script=sci_arttext\&pid=S230 7-21132017000300005

4. Pessoa-Giasanti-Tavares AR, Taboada-Sobral AP, JansiskiMotta L. Uso de la aplicación WhatsApp por estudiantes de Odontología de Sao Paulo, Brasil. Revista Cubana de Información en Ciencias de la Salud. 2016;27(4):503-514. Disponible

en: http://scielo.sld.cu/scielo.php?script=sci_arttext\&pid=S230 7-21132016000400007

5. Padrón C, Bravo M. Competencias TIC para la gestión del conocimiento: un aporte desde el modelo TPACK. Educare. 2014;18(3):49-73. Disponible https://dialnet.unirioja.es/servlet/articulo? codigo $=535868$ 3

6. Santamaría G, Hernández E, Suárez F. Aplicaciones de salud para móviles: Uso en pacientes de Medicina Interna en el Hospital Regional de Duitama, Boyacá, Colombia. Revista Cubana de Información en Ciencias de la Salud. 2016;27(3):271-285. Disponible en: http://scielo.sld.cu/scielo.php?script=sci_arttext\&pid=S230 7-21132016000300002

7. Martínez J, Harris J, Fortich N, Plazas J. Prevalencia de lesiones orales y su distribución en la cavidad bucal. Revista Ciencia y Salud Virtual. 2014;6(2):92-98. DOI $10.22519 / 21455333.420$

8. Rodríguez M, Portillo A, Lama E, Hernández S. Lesiones bucales asociadas con el uso de prótesis en pacientes de la comunidad de Kantunil, Yucatán. Revista ADM. 2014;71(5):221-225. Disponible en: https://www.medigraphic.com/pdfs/adm/od2014/od145d.pdf
9. Tavares A, Caetano A, Monken de Assis S, Jansiski L. Uso de una aplicación para registrar información sobre pacientes estomatológicos desde la perspectiva de los cirujanos dentales. Revista Cubana de Información en Ciencias de la Salud. 2018;29(1):41-54. Disponible en: http://scielo.sld.cu/scielo.php?script=sci_arttext\&pid=S230 7-21132018000100004

10. Díaz M, Martin C, Somacarrera ML. Uso de dispositivos móviles en el aula. Oralmedx, una "app" de ayuda al diagnóstico de lesiones orales. JIIU. 2014;11(116):221-227. Disponible en: http://docplayer.es/9930320-Uso-dedispositivos-moviles-en-el-aula-oralmedx-una-app-deayuda-al-diagnostico-de-lesiones-orales.html

11. Mauro I, González M, Collado L. Aplicaciones móviles en nutrición, dietética y hábitos saludables: análisis y consecuencia de una tendencia a la alza. Nutrición Hospitalaria. http://dx.doi.org/10.3305/nh.2014.30.1.7398

12. Peirats J, Marín D, Granados J, Morote D. Competencia digital en los planes de estudios de universidades públicas españolas. REDU: Revista de Docencia Universitaria. 2018;16(1):175-192. Disponible en: https://dialnet.unirioja.es/servlet/articulo?codigo $=651132$ 4

13. Arriaga 0 , González M, Ramírez A, Iriarte A, López L, Arciniega A. Uso de aplicaciones de la web 2.0 para la evaluación del aprendizaje significativo. Pistas Educativas. 2018; 40(130):284-301. Disponible en: http://www.itcelaya.edu.mx/ojs/index.php/pistas/article/ view/1704/1349

14. Escobar H, Benavides L. Objetos virtuales de aprendizaje y un laboratorio virtual de química en la enseñanza de la ley de conservación de la masa. Historia de la Educación Colombiana. 2015;18(18):169-200. Disponible en: https://dialnet.unirioja.es/servlet/articulo? codigo $=677832$ 7

15. Salgado N, Sánchez H, Rico M. Evaluación de la metodología y prácticas educativas mediante el uso de plataformas virtuales para el aprendizaje del inglés. Análisis de caso del instituto de idiomas de la Universidad Tecnológica Equinoccial de la ciudad de Quito. Revista TecnológicaESPOL. 2015;28(5): 8-26. Disponible en: http://www.rte.espol.edu.ec/index.php/tecnologica/article /view/422/288

16. Del Vasto H. Influencia de las tecnologías de información y comunicación (TIC) en el proceso enseñanza-aprendizaje: una mejora de las competencias digitales. Rev Cient Gnall José María Córdova. 2015;13(16):121-132. Disponible en: http://www.scielo.org.co/pdf/recig/v13n16/v13n16a07.pd $\mathrm{f}$

17. Parra-Esquivel E, Peñas-Felizzola O, Gómez-Galindo A. Objetos virtuales para el aprendizaje autorregulado de estudiantes de terapia ocupacional. Revista de Salud Pública. 2017;19(6): 760-765. http://dx.doi.org/10.15446/rsap.v19n6.62966

18. Córdoba M, López E, Ospina J, Polo J. Estudiantes de la básica y media con respecto al uso de las TIC como herramientas de apoyo a su aprendizaje. Trilogía Ciencia Tecnología Sociedad. 2016;9(16):113-125. Disponible en: https://dialnet.unirioja.es/servlet/articulo?codigo=576349 3 